\title{
Enfrentamento a covid-19: importância da educação permanente em serviços de saúde
}

\author{
Coping with covid-19: importance of continuing education in health services
}

Cómo hacer frente al covid-19: importancia de la educación continua en los servicios de salud

José Luís Silva dos Santos ${ }^{1 *}$, Filipe Almeida de Santana ${ }^{2}$, Clarice Souza Serafim¹, Lucas Ribeiro de Freitas ${ }^{1}$, Wenderson Laelcio da Silva Oliveira ${ }^{1}$, Marcos Vinicius Santana de Melo ${ }^{1}$, Douglas Henrique Da Silva Ferreira ${ }^{1}$, Wanuska Munique Portugal ${ }^{1}$, Lenio José de Pontes Costa $^{1}$, Giselda Bezerra Correia Neves ${ }^{1}$.

\section{RESUMO}

Objetivo: Identificar na literatura quais são as evidências científicas sobre a importância da Educação Permanente em Saúde (EPS) como estratégias de enfrentamento a pandemia da COVID-19. Métodos: Revisão integrativa desenvolvida a partir de publicações, entre os anos de 2019 a 2021, nas bases de dados Medical Literature Análise e Retrieval System on-line (MEDLINE), Literatura Latino-Americana e do Caribe em Ciências da Saúde (LILACS), Banco de Dados em Enfermagem (BDENF) e Índice Bibliográfico Espanhol em Ciências da Saúde (IBECS) através da Biblioteca Virtual em Saúde (BVS), além de levantamento bibliográfico na base de dados da Acervo+. Resultados: Encontraram-se 91 artigos nas bases de dados, sendo selecionados 17 artigos para compor a revisão integrativa, tendo como predominância de estudos relatos de experiência, dos quais em sua maioria apresentavam estratégias de intervenções no combate à COVID-19. Considerações finais: A alta transmissibilidade da SARS-CoV-2 apresenta-se como um desafio para a saúde pública, com significativos impactos na economia mundial, e nos sistemas de saúde, através das ações educativas tais como Higienização das mãos; Paramentação e Desparamentação; Simulação clínica; Uso de tecnologias digitais entre outros. Diante deste cenário pandêmico a EPS é uma ferramenta imprescindível no enfrentamento a pandemia da COVID-19.

Palavras-chave: Educação em saúde, Educação permanente, COVID-19.

\begin{abstract}
Objective: To identify in the literature what is the scientific evidence on the importance of Permanent Health Education (PHE) strategies to cope with the COVID-19 pandemic. Methods: Integrative review developed from publications, between 2019 and 2021, in databases Medical Literature Analysis and Retrieval System Online (MEDLINE), Latin American and Caribbean Literature on Health Sciences (LILACS), Nursing Database (BDENF), and Spanish Bibliographic Index in Health Sciences (IBECS) through the Virtual Health Library (VHL), in addition to bibliographic survey in the Acervo+ database. The Documentary review was included in digital platforms and institutional websites of the Ministry of Health, with publications from 2019 to 2021. Results: We found 91 articles in the databases, and 17 articles were selected to make up the integrative review, with experience reports as a predominance of studies, most of which presented intervention strategies to combat COVID-19. Final considerations: The high transmissibility of SARS-CoV-2 presents itself as a challenge for public health, with significant impacts on the world economy, and on health systems, through educational actions such as hand hygiene; Paramentation and Deparamentation; Clinical simulation; Use of digital technologies among others. Given this pandemic scenario, PHE is an indispensable tool in coping with the COVID-19 pandemic.
\end{abstract}

Keywords: Health education, Continuing education, COVID-19.

${ }^{1}$ Centro Universitário Brasileiro (UNIBRA), Recife - PE. *E-mail: jose.luiss16@hotmail.com

2 Unidade de Apoio ao Diabético e Oxigenoterapia Hiperbárica (UNIAD), Caruaru - PE.

SUBMETIDO EM: 8/2021

ACEITO EM: 8/2021

PUBLICADO EM: 8/2021 


\section{RESUMEN}

Objetivo: Identificar en la literatura cuál es la evidencia científica sobre la importancia de la Educación Permanente para la Salud (EPS) estrategias para hacer frente a la pandemia de COVID-19. Métodos: Revisión integradora desarrollada a partir de publicaciones, entre 2019 y 2021, en bases de datos Medical Literature Analysis and Retrieval System en línea (MEDLINE), Latin American and Caribbean Literature on Health Sciences (LILACS), Base de Datos de Enfermería (BDENF) e Índice Bibliográfico Español en Ciencias de la Salud (IBECS) a través de la Biblioteca Virtual en Salud (BVS), además de la encuesta bibliográfica en la base de datos de la Acervo+. La revisión documental se incluyó en plataformas digitales y sitios web institucionales del Ministerio de Salud, con publicaciones de 2019 a 2021. Resultados: Se encontraron 91 artículos en las bases de datos, y se seleccionaron 17 artículos para la revisión integradora, con predominio de estudios informes de experiencia, de los cuales la mayoría de ellos tenían estrategias de intervención para combatir el COVID-19. Consideraciones finales: La alta transmisibilidad del SARS-CoV-2 se presenta como un desafío para la salud pública, con impactos significativos en la economía mundial, y en los sistemas de salud, a través de acciones educativas como la higiene de manos; Paramentación y Deparamentación; Simulación clínica; Uso de tecnologías digitales entre otros. Ante este escenario pandémico, la EPS es una herramienta indispensable para hacer frente a la pandemia de COVID-19.

Palabras clave: Educación para la salud, Educación continua, COVID-19.

\section{INTRODUÇÃO}

Pesquisadores chineses informam à Organização Mundial de Saúde (OMS) que foram identificados no mês de dezembro de 2019, casos de pneumonias virais com origem desconhecida na cidade de Wuhan, República popular da China. A rotina de vigilância, instituída no local, permitiu que as análises desses pacientes evidenciassem uma nova variante do coronavírus, inicialmente nomeado 2019-nCoV (novo coronavírus 2019) e, posteriormente, substituído para SARS-CoV-2 (Coronavírus 2 da Síndrome Respiratória Aguda Grave), causador da COVID-19 (Doença do Coronavírus em 2019). Um vírus com alta transmissibilidade e morbimortalidade, sendo o primeiro caso identificado no Brasil em 26 de fevereiro de 2020, no estado de São Paulo (MOTTA DL, et al., 2020).

O coronavírus pertence a uma grande família de vírus, sendo comuns em diferentes espécies de animais (morcegos, camelos, gado e gatos), atualmente, o que se sabe é que o novo coronavírus possui uma alta e sustentada transmissibilidade entre as pessoas, sendo assim, faz se necessário a providência de planos de contingência que promovam as medidas de prevenção e controle, visando a redução significativa de transmissibilidade (SILVA GPC, et al., 2020).

O SARS-CoV-2 tem mostrado comportamento incomum, com alta letalidade, sendo transmitido pelo contato entre pessoas por meio de aerossóis respiratórios e contato indireto com objetos e superfícies contaminadas. A transmissibilidade viral se dá através das mucosas e as manifestações clínicas, podem se apresentar de diversas formas, desde um simples resfriado até a Síndrome Respiratória Aguda Grave (SRAG). Os sintomas clínicos mais frequentes são febre, tosse seca e dispneia, associado a anosmia (perda do olfato), disgeusia (perda do paladar) (MINISTÉRIO DA SAÚDE, 2020).

O Ministério da Saúde reassume que a Educação Permanente em Saúde (EPS) deve se configurar na prática como uma aprendizagem pelo trabalho de maneira que os processos de ensino e aprendizagem sejam incorporados ao cotidiano dos serviços de saúde por meio da aprendizagem significativa, com vistas na transformação das práticas profissionais. A política de EPS se apresenta historicamente como potencial para impulsionar processos de mudança e consolidação do modelo de assistência à saúde, uma vez que seu objetivo é direcionado para a incorporação, por parte dos trabalhadores, de processos de trabalhos condizentes com os princípios e diretrizes do SUS, que reconhece em seu ordenamento jurídico, por meio da Lei 8.080 da Constituição de 1988, a responsabilidade e a competência para formação dos trabalhadores da saúde como estratégia de consolidação do modelo assistencial do SUS (BARBOSA SIM e MOREIRA FJF, 2019).

Para Martins AB, et al. (2020), Devido as situações atípicas para muitas relações de saúde-doença trazida pela Covid-19 foi possível identificar a importância de estabelecer um cuidado mais humanizado aos 
profissionais, usuários e familiares. Compreende-se que o desenvolvimento da humanização perpassa pelo acolhimento das pessoas que estão em adoecimento, dos familiares fragilizados pela doença e pelo distanciamento, onde os seus entes encontram-se internados em unidades restritas para a assistência à saúde com medidas impostas para redução dos riscos de contaminação.

A Política Nacional de Humanização (PNH) apresenta como preceito a inclusão de trabalhadores, usuários e gestores na produção e gestão do cuidado e dos processos de trabalho, objetivando modificar relações de trabalho a partir do contato e da comunicação entre as pessoas e grupos. Dentre os propósitos da PNH incluem a formação e intervenção por meio de cursos e oficinas partindo de pontos discutidos nos processos de trabalho, implicando em mudanças no cotidiano dos serviços de saúde (MINISTÉRIO DA SAÚDE, 2013).

A Política Nacional de Educação Permanente em Saúde (PNEPS) e a Política Nacional de Humanização $(\mathrm{PNH})$ dialogam à medida em que procuram transformar as práticas no ambiente de trabalho. Para ambas a modificação do processo de trabalho acontece a partir do protagonismo de diversos atores do sistema de saúde: trabalhadores, gestores e usuários, os quais estão diretamente implicados no processo de construção da saúde. Assim, existe uma complementariedade dessas políticas na construção do cuidado e reformulação das práticas (LOPES RSTM, et al., 2019).

A PNEPS objetiva a melhoria nas práticas formativas e no desenvolvimento dos trabalhadores para que estes atuem efetivamente no sistema público de saúde, a PNH estabelece diretrizes, dispositivos e ferramentas que propiciem a operacionalidade das práticas humanizadoras. Por isso, a PNEPS mostra-se como um dos mais potentes instrumentos na garantia da força de trabalho estando voltada para o cuidado humanizado nos serviços de saúde (LOPES RSTM, et al., 2019).

Sendo assim o objetivo deste trabalho de revisão foi buscar informações acerca da importância da Educação Permanente em Saúde como estratégias de enfrentamento a pandemia da COVID-19.

\section{MÉTODOS}

Trata-se de uma revisão integrativa, a estratégia de identificação e seleção dos estudos foi a busca de publicações indexadas nas bases de dados Medical Literature Análise e Retrieval System on-line (MEDLINE), Literatura Latino-Americana e do Caribe em Ciências da Saúde (LILACS), Banco de Dados em Enfermagem (BDENF) e Índice Bibliográfico Espanhol em Ciências da Saúde (IBECS) através da Biblioteca Virtual em Saúde (BVS), além de levantamento bibliográfico na base de dados da Acervo+.

Incluiu-se revisão documental em plataformas digitais e sites institucionais, tais quais o Ministério da Saúde (MS), com publicações dos anos de 2019 e 2021, elencou-se os artigos oriundos de pesquisas, que estavam disponíveis online e gratuitamente. Para isso, seguiu-se os seis passos para seu desenvolvimento: formulação da questão norteadora; busca na literatura; extração dos dados dos estudos selecionados; avaliação dos estudos; interpretação e síntese dos resultados; apresentação da revisão integrativa.

Para responder ao objetivo deste estudo, formulou-se a seguinte questão norteadora "Qual a importância da educação permanente em saúde e quais estratégias podem ser utilizadas no enfrentamento a pandemia da COVID-19?".

A busca de literatura envolveu todas as bases de dados disponíveis na Biblioteca Virtual em Saúde (BVS) e os descritores utilizados foram "Educação em Saúde" and "Educação Permanente" and "COVID-19", foram utilizadas no campo "título, resumo, assunto", sendo a coleta de dados realizada no mês de maio a julho de 2021. Enquanto critérios de inclusão, foram elencados estudos disponibilizados na íntegra em língua Portuguesa, Inglês e Espanhol produzidos nos anos 2019 a 2021. Foram excluídas da amostra artigos duplicados, artigos de revisão, reflexão/debates, comentários, editorias e cartas.

\section{RESULTADOS}

Como resultado da busca, foram obtidos 91 artigos científicos, sendo que 36 não respondiam aos objetivos do trabalho, 4 não estavam disponíveis na íntegra, 1 aparecia de forma duplicada e 33 foram excluídos após a leitura de título e resumo. Por fim, estabeleceu-se a amostra de 17 pesquisas selecionadas para análise e discussão dos dados, de acordo com o fluxograma explicativo apresentado (Figura 1). 
Figura 1 - Fluxograma das etapas para seleção dos artigos desta revisão integrativa.

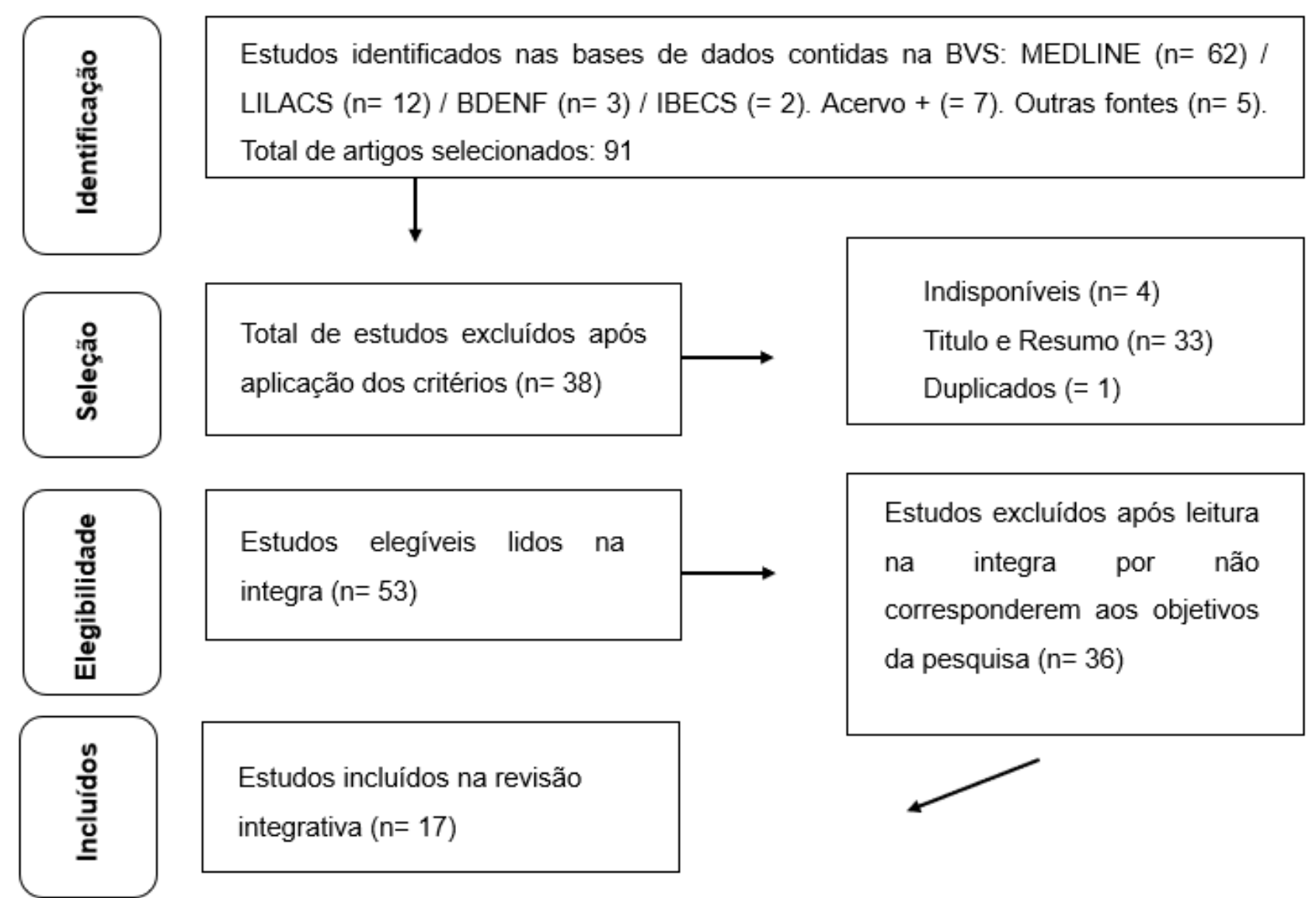

Fonte: Santos JLS, et al., 2021.

Com intuito de concentrar, integralizar/sistematizar os achados desta revisão, foi constuído um quadro Sínte (Quadro 1). 


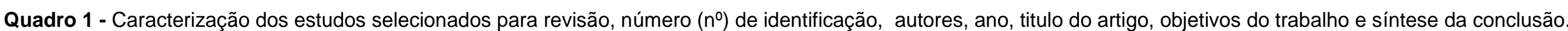

\begin{tabular}{|c|c|c|c|c|c|}
\hline $\mathbf{N}$ & Autores(Ano) & Título & Tipo de estudo & Objetivo (Amostra) & Síntese dasconclusões \\
\hline 1 & ZINGRA KN, et al. (2020) & $\begin{array}{l}\text { Educação permanente para } \\
\text { profissionais da área da saúde } \\
\text { como estratégia de combate ao } \\
\text { enfretamento da pandemia de } \\
\text { COVID-19 na região norte: relato } \\
\text { de experiência. }\end{array}$ & Relato de experiência & $\begin{array}{l}\text { Descrever o processo de capacitação à } \\
\text { profissionais da saúde como forma de } \\
\text { estratégia de combate ao enfrentamento da } \\
\text { pandemia de covid-19 em municípios do } \\
\text { Estado de Rondônia e do Amazonas. }\end{array}$ & $\begin{array}{l}\text { Tal processo, pode ser um facilitador para } \\
\text { melhorar a estratégia de cuidado e integrar de } \\
\text { forma harmônica as equipes que estariam na } \\
\text { linha de frente ao combate do covid-19. }\end{array}$ \\
\hline 2 & NETO JBSB, et al. (2020) & $\begin{array}{l}\text { Construção de tecnologias } \\
\text { educativas como forma de } \\
\text { educação em saúde para a } \\
\text { prevenção da Covid-19: relato de } \\
\text { experiência. }\end{array}$ & Relato de experiência & $\begin{array}{l}\text { Relatar a construção de duas tecnologias } \\
\text { educacionais com o tema de "Orientações } \\
\text { para a limpeza terminal de transportes } \\
\text { coletivos e individuais e uso de Equipamentos } \\
\text { de Proteção Individual. }\end{array}$ & $\begin{array}{l}\text { As tecnologias educacionais virtuais } \\
\text { elaboradas são de suma importância para o } \\
\text { combate da pandemia de covid- } 19 \text {. }\end{array}$ \\
\hline 3 & SANTOS RLN, et al. (2021) & $\begin{array}{l}\text { Potencialidade da Educação } \\
\text { Permanente na prevenção da } \\
\text { infecção pelo Covid-19 em } \\
\text { profissionais de saúde: relato de } \\
\text { experiência. }\end{array}$ & Relato de experiência & $\begin{array}{l}\text { Relatar a experiência de treinamentos } \\
\text { realizados sobre as medidas preventivas } \\
\text { adotadas durante a pandemia do COVID-19, } \\
\text { visando o fortalecimento e empoderamento } \\
\text { por parte dos profissionais. }\end{array}$ & $\begin{array}{l}\text { Acreditar na importância do trabalho realizado } \\
\text { pela educação permanente é perceber que é } \\
\text { possível educar, de forma humanizada. }\end{array}$ \\
\hline 4 & $\begin{array}{l}\text { GONÇALVES SO, et al. } \\
(2020)\end{array}$ & $\begin{array}{l}\text { Programa } \quad \text { de } \\
\text { permanente em saúde e a praxis } \\
\text { profissional: } \\
\text { desafios. }\end{array}$ & $\begin{array}{l}\text { Pesquisa descritiva, } \\
\text { quantiqualitativa }\end{array}$ & $\begin{array}{l}\text { ldentificar o conhecimento dos profissionais } \\
\text { da atenção primária a saúde sobre a Política } \\
\text { Nacional de Educação Permanente em } \\
\text { Saúde. }\end{array}$ & $\begin{array}{l}\text { O estudo identificou o conhecimento dos } \\
\text { gestores e dos profissionais da Atenção } \\
\text { Primária à sobre a PNEPS e EPS. }\end{array}$ \\
\hline 5 & JUNIOR AMF, et al. (2021) & $\begin{array}{l}\text { COVID-19 em profissionais da } \\
\text { saúde, vivências e perspectivas: } \\
\text { um relato de experiência. }\end{array}$ & Relato de experiência & $\begin{array}{l}\text { Relatar a experiência de profissionais da área } \\
\text { da saúde que trabalhavam em um hospital } \\
\text { público no combate ao COVID-19. }\end{array}$ & $\begin{array}{l}\text { Grande parcela dos profissionais foi exposta a } \\
\text { diversos riscos ocupacionais, principalmente, } \\
\text { ao estresse ocupacional, tal risco modificou a } \\
\text { rotina de trabalho e até o relacionamento } \\
\text { interpessoal fora dos hospitais. }\end{array}$ \\
\hline 6 & MARTINS AB, et al. (2020) & $\begin{array}{l}\text { A assistência multiprofissional a } \\
\text { pacientes em tratamento de } \\
\text { COVID-19 e a minimização do } \\
\text { distanciamento familiar em um } \\
\text { serviço de pronto atendimento em } \\
\text { Manaus, Amazonas. }\end{array}$ & Relato de experiência & $\begin{array}{l}\text { Relatar a assistência multiprofissional aos } \\
\text { pacientes em tratamento de COVID-19 com } \\
\text { vistas à minimização do distanciamento } \\
\text { familiar em um serviço de pronto atendimento } \\
\text { em Manaus. }\end{array}$ & $\begin{array}{l}\text { As medidas realizadas foram de grande } \\
\text { importância para atenuar os impactos do } \\
\text { distanciamento familiar dos pacientes e } \\
\text { enfrentamento à doença. }\end{array}$ \\
\hline 7 & SILVA JF, et al. (2020) & $\begin{array}{l}\text { Educação permanente em saúde } \\
\text { no contexto de uma unidade de } \\
\text { saúde da família no município do } \\
\text { Rio de Janeiro, Brasil. }\end{array}$ & $\begin{array}{l}\text { Pesquisa de campo, } \\
\text { de natureza qualitativa }\end{array}$ & $\begin{array}{l}\text { Analisar a percepção dos profissionais sobre } \\
\text { as práticas de Educação Permanente em } \\
\text { Saúde, considerando a relação entre a prática } \\
\text { profissional e a produção de saberes. }\end{array}$ & $\begin{array}{l}\text { Embora existam políticas indutoras que } \\
\text { orientam a Educação Permanente em Saúde, } \\
\text { a efetividade do seu potencial para produção } \\
\text { de saberes na prática dos profissionais que } \\
\text { atuam na Saúde da Família permanece como } \\
\text { incipiente. }\end{array}$ \\
\hline 8 & $\begin{array}{l}\text { FERREIRA CHP, et al. } \\
(2020)\end{array}$ & $\begin{array}{l}\text { Estratégias adotadas no } \\
\text { enfrentamento da covid-19: Relato } \\
\text { de experiência profissional. }\end{array}$ & $\begin{array}{l}\text { Estudo descritivo, do } \\
\text { tipo relato de } \\
\text { experiência }\end{array}$ & $\begin{array}{l}\text { Relatar a experiênciaacerca da estruturação } \\
\text { das ações adotadas no enfrentamento a } \\
\text { pandemia da Covid-19. }\end{array}$ & $\begin{array}{l}\text { Enfatiza-se diante dapandemia a relevância } \\
\text { da manutenção de contínua atualização } \\
\text { técnico científica. }\end{array}$ \\
\hline
\end{tabular}




\begin{tabular}{|c|c|c|c|c|c|}
\hline $\mathbf{N}$ & Autores(Ano) & Título & Tipo de estudo & Objetivo (Amostra) & Síntese dasconclusões \\
\hline 9 & SILVA GPC, et al. (2020) & $\begin{array}{l}\text { Atividades educativas para uso } \\
\text { adequado de equipamentos de } \\
\text { proteção individual em hospital } \\
\text { federal de referência. }\end{array}$ & $\begin{array}{l}\text { Estudo descritivo, do } \\
\text { tipo relato de } \\
\text { experiência }\end{array}$ & $\begin{array}{l}\text { Relatar a experiência da realização de } \\
\text { atividades educativas sobre Paramentação e } \\
\text { Desparamentação de Equipamentos de } \\
\text { Proteção Individual (EPI). }\end{array}$ & $\begin{array}{l}\text { As atividades possibilitaram um cuidado da } \\
\text { instituição com os seus colaboradores, da } \\
\text { equipe de enfermagem e multiprofissional. }\end{array}$ \\
\hline 10 & RIOS MFA, et al. (2020) & $\begin{array}{l}\text { Atenção primáriaà saúde frente à } \\
\text { covid-19 em um centro de saúde. }\end{array}$ & Relato de experiência & $\begin{array}{l}\text { Relatar as estratégias de enfrentamento à } \\
\text { COVID-19 de um Centro de Saúde daAtenção } \\
\text { Primária à Saúde de um município do sul da } \\
\text { Bahia. }\end{array}$ & $\begin{array}{l}\text { O profissional de Enfermagem é ó } \\
\text { protagonista da Atenção Primária à Saúde, } \\
\text { destacando- se desde o planejamento às } \\
\text { execuções e avaliação das ações } \\
\text { implementadas. }\end{array}$ \\
\hline 11 & $\begin{array}{c}\text { OLIVEIRA FF e } \\
\text { HONORATO KA (2021) }\end{array}$ & $\begin{array}{l}\text { Atividade lúdica e educativa para } \\
\text { higienização dasmãos em tempos } \\
\text { de } \\
\text { pandemia: relatode experiência. }\end{array}$ & $\begin{array}{c}\text { Relato de experiência } \\
\text { profissional }\end{array}$ & $\begin{array}{l}\text { Descrever a experiência de uma atividade } \\
\text { lúdica parareflexão da técnica de higienização } \\
\text { das mãos entre profissionais de enfermagem } \\
\text { frente ao atendimento de paciente com } \\
\text { Coronavírus. }\end{array}$ & $\begin{array}{l}\text { A educação permanente é primordial para } \\
\text { incentivar e realizar as técnicas adequadas, } \\
\text { principalmente as que se referem às barreiras } \\
\text { de exposição ao vírus. }\end{array}$ \\
\hline 12 & $\begin{array}{l}\text { SERRAVALLE LMK, et al. } \\
(2021)\end{array}$ & $\begin{array}{l}\text { Incorporação de tecnologias } \\
\text { digitais nas estratégias de apoio à } \\
\text { RENAST-BA durante a pandemia } \\
\text { da covid-19. }\end{array}$ & Relato de experiência & $\begin{array}{l}\text { Descrever a incorporação de tecnologias } \\
\text { digitais no desenvolvimento do apoio } \\
\text { institucional e técnico e das atividades de } \\
\text { educação permanente pelo Cesat/Divast. }\end{array}$ & $\begin{array}{l}\text { Reorganizou os processos de trabalho, com a } \\
\text { substituição das atividades presenciais pela } \\
\text { utilização de ferramentas digitais, de modo a } \\
\text { evitar aglomerações. }\end{array}$ \\
\hline 13 & $\begin{array}{l}\text { BRANCO AO E TAVARES } \\
\text { MMC (2020) }\end{array}$ & 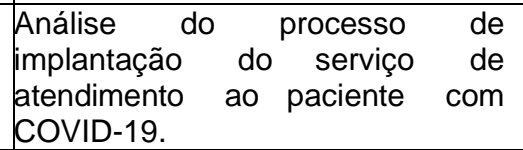 & $\begin{array}{l}\text { Relatório de } \\
\text { experiência } \\
\text { sistemática }\end{array}$ & $\begin{array}{l}\text { Analisar o processo de planejamento e } \\
\text { implantação de um serviço de atendimento ao } \\
\text { paciente com COVID-19 em um hospital } \\
\text { universitário. }\end{array}$ & $\begin{array}{l}\text { É importante utilizar processos de trabalho e } \\
\text { Educação Continuada em Saúde como } \\
\text { ferramentas para orientar o cuidado aos } \\
\text { pacientes COVID-19. }\end{array}$ \\
\hline 14 & MOTTA DL, et al. (2020) & $\begin{array}{l}\text { COVID-19 evidências para todos: } \\
\text { desenvolvimento de um objeto de } \\
\text { aprendizagem no ensino em saúde. }\end{array}$ & Relato de experiência & $\begin{array}{l}\text { Descrever o desenvolvimento de um objeto de } \\
\text { aprendizagem focado em evidências } \\
\text { científicas sobre o COVID-19. }\end{array}$ & $\begin{array}{l}\text { O recurso permite uma rápida consulta das } \\
\text { melhores recomendações científicas. }\end{array}$ \\
\hline 15 & $\begin{array}{l}\text { PIMENTÃO RA, et al. } \\
(2021)\end{array}$ & $\begin{array}{lcr}\text { Simulação } & \text { clínica } & \text { para } \\
\text { enfrentamento } & \text { da } & \text { covid-19: } \\
\text { treinamento } & \text { complementar de } \\
\text { enfermeiros. } & & \end{array}$ & Relato de experiência & $\begin{array}{l}\text { Relatar a experiência da construção e do } \\
\text { desenvolvimento da simulação clínica como } \\
\text { ferramenta pedagógica para a formação } \\
\text { complementar de enfermeiros no } \\
\text { enfrentamento à COVID-19. }\end{array}$ & $\begin{array}{l}\text { A simulação clínica facilitou o } \\
\text { desenvolvimento do pensamento crítico, da } \\
\text { comunicação entre profissional e paciente, } \\
\text { das habilidades técnicas, afetivas. }\end{array}$ \\
\hline 16 & GEREMIA SD, et al. (2020) & $\begin{array}{l}200 \text { Anos de Florence e os desafios } \\
\text { da gestão das práticas de } \\
\text { enfermagem na pandemia COVID- } \\
19 .\end{array}$ & Estudo qualitativo & $\begin{array}{l}\text { Analisar os principais desafios da enfermagem } \\
\text { no enfrentamento a COVID-19 sob a } \\
\text { perspectiva de enfermeiros gestores. }\end{array}$ & $\begin{array}{l}\text { A situação de pandemia tem elevado a } \\
\text { enfermagem a uma posição de protagonismo } \\
\text { prático e científico pela proatividade e } \\
\text { capacidade de liderança. }\end{array}$ \\
\hline 17 & LOPES RSTM, et al. (2019) & $\begin{array}{l}\text { Educação permanente e } \\
\text { humanização na transformação das } \\
\text { práticas na atenção básica. }\end{array}$ & Estudo qualitativo & $\begin{array}{l}\text { Avaliar os processos de organização e } \\
\text { implantação da humanização segundo a } \\
\text { Política Nacional de Educação Permanente } \\
\text { em Saúde na Atenção Básica. }\end{array}$ & $\begin{array}{l}\text { As Políticas de Educação Permanente e de } \\
\text { Humanização têm influenciado de forma } \\
\text { positiva os processos de trabalho das equipes } \\
\text { de saúde na atenção básica. }\end{array}$ \\
\hline
\end{tabular}

Fonte: Santos JLS, et al., 2021.

REAEnf | Vol.13 | DOI: https://doi.org/10.25248/REAEnf.e8669.2021 $\quad$ Página $\mathbf{6}$ de $\mathbf{1 0}$




\section{DISCUSSÃO}

No dia 3 de fevereiro de 2020 é declarado no Brasil, Emergência em Saúde Pública de Importância Nacional (ESPIN), em decorrência da pandemia da covid-19, imediatamente iniciou-se as ações de vigilância em saúde, com o objetivo de reduzir o número de casos novos, foram instituídas medidas de prevenção, tais quais reorganização de processos de trabalho, distanciamento social, etiqueta respiratória, uso de máscaras e higienização das mãos (SERRAVALLE LMK, et al., 2021).

A Covid-19 apresenta amplo espectro clínico podendo os portadores serem assintomáticos, oligossintomáticos ou manifestarem quadros graves como a Síndrome Respiratória Aguda Grave (SRAG), especialmente em grupos de risco, nos quais se enquadram pacientes idosos, gestantes, imunodeprimidos e com comorbidades, a exemplo do diabetes mellitus e portadores de doenças cardíacas crônicas (MARTINS $A B$, et al., 2020).

A educação sobre a temática da COVID-19 é fundamental, tanto para os profissionais de saúde quanto para a população principalmente em um momento onde o controle na disseminação do vírus, é necessário. Estratégias educativas em saúde são métodos que devem ser utilizados para informar e influenciar as decisões individuais e coletivas, portanto, importantes para promover medidas de prevenção. $O$ acelerado processo de atualização do conhecimento acerca da COVID-19 exige dos profissionais na linha de frente a devida atualização de forma permanente com a finalidade de torna-los aptos a desenvolverem uma prática de assistência ampla e segura ao paciente e toda a sociedade (MOTTA DL, et al., 2020; FERREIRA CHP, et al., 2020).

As mudanças causadas pelo novo coronavírus resultaram em intervenções numerosas que modificaram significativamente o dia a dia da população colocando em risco os profissionais de saúde. Ao atuar na linha de frente, nesse contexto, a equipe de enfermagem tem lidado com maior índice de contaminação e óbitos, além de alta incidência de doenças psicossomáticas. A partir desses eventos, ampliam-se os debates que envolvem a capacitação dos serviços de saúde (GEREMIA SD, et al., 2020).

De acordo com o estudo de Junior AMF, et al. (2021), os profissionais envolvidos na assistência ao paciente com COVID-19 relataram que o dimensionamento de informações sobre a doença e o número de óbitos no Brasil gerava uma sensação de fragilidade e, muitas vezes, causavam reações psicossomáticas que se assimilavam com os sinais e sintomas da COVID-19, tal fato, gerou crises de ansiedade que, por vezes, interferiram diretamente na prática do cuidado aos pacientes.

A Educação em saúde é identificada como uma estratégia que tem potencial para prevenir e promover a saúde em diversas áreas de atuação. Diante de um cenário pandêmico, essa estratégia tem sido priorizada e instituida como prioridade entre as tarefas de trabalho a fim de promover a saúde e prevenir a COVID-19 (RIOS MFA, et al., 2020).

A Educação Permanente em Saúde é uma proposta ético-político-pedagógica que objetiva modificar e qualificar a atenção em saúde por meio de estratégias que viabilize os processos de formação, as ações de educação em saúde e também favorecer a organização das atividades e dos serviços numa concepção multiprofissional e intersetorial. Em 2004, por meio da Portaria №198 de fevereiro de 2004, o Ministério da Saúde (MS) preconizou a PNEPS como uma estratégia do Sistema Único de Saúde voltada para a formação e o desenvolvimento dos seus profissionais, com objetivo de promover a articulação da integração ensinoserviço e comunidade, reconhecendoa regionalização da gestão do SUS, como alicerce para o desenvolvimento de iniciativas direcionadas ao enfrentamento das necessidades e entraves do sistema (SILVA GPC, et al., 2020).

A EPS procura transformar as práticas em saúde, para que isso ocorra de forma eficaz é necessário que os profissionais identifiquem e sinalizem algumas necessidades de saúde da população. Através dessa perspectiva busca-se soluções de forma conjunta para os desafios e dificuldades encontradas. No entanto, muitos profissionais percebem as instituições de educação e o Sistema Único de Saúde (SUS) como únicos responsáveis pela problematização do trabalho e das organizações (RIOS MFA, et al., 2020). 
Segundo Silva JF, et al. (2020) afirma que a EPS deve estar em consonância com os acontecimentos cotidianos, tanto do ambiente de trabalho quanto da vida dos indivíduos. Entretanto o mundo do trabalho está relacionado com o lugar ocupado pelos trabalhadores e estes são os atores principais da construção do trabalho. A educação em saúde deve estar direcionada para a transformação das práticas e focada no desenvolvimento de competências que dialoguem com as reais necessidades dos serviços. A análise constante dos profissionais e das instituições devem ser realizadas visando identificar possíveis propostas de intervenção.

Com o aumento crescente do número de casos por COVID-19, observou-se mudanças significativas na rotina de trabalho nos serviços de saúde, evidenciado por falta de leitos, aumento de internações hospitalares e consequentemente a superlotação das unidades e falta de equipamentos, desta forma impactando na saúde dos profissionais que estão na assistência (FERREIRA CHP, et al., 2020).

A COVID-19 tem um potencial para sobrecarrega o setor saúde, é necessário a participação de todo o sistema de saúde, municipal, regional, estadual e federal, além da participação social, afim de promover a redução de impactos socioeconômicos, morbidade e mortalidade, impostas pela pandemia da covid-19 (MINISTÉRIO DA SAÚDE, 2020).

O insólito cenário da pandemia do Coronavírus, exigiu que o sistema saúde mundial apresentasse aceleradas respostas ao enfrentamento da doença. Tanto no âmbito científico quanto no comportamental da população, ambos preocupados na necessidade de reorganizar o sistema e as boas práticas sanitárias para assim diminuir a trasmissibilidade do vírus (MONTALVÃO SA, et al., 2020).

A implementação de um programa de EPS traz benefícios para a equipe de saúde propiciando discussão entre o grupo de trabalho. Assim, importante destacar que, para tanto, faz-se necessário um despertar dos profissionais e principalmente da gestão, que conduza a estruturação de um programa que possibilite a capacitação do grupo de trabalho. Além disso, traz o protagonismo da equipe diante de seu usuário, a fim de propiciar melhor qualidade de assistência (GONÇALVES SO, et al., (2020).

\section{A educação na saúde como estratégia de enfrentamento a pandemia da COVID-19 e suas contribuições para prática:}

Diante da apreensão em capacitar a equipe multidisciplinar, algumas instituições de saúde implementaram ações de educação permanente, visando a prevenção dos profissionais, por meio da execução de treinamentos realizados sobre as medidas preventivas adotadas durante a pandemia do COVID-19, visando o fortalecimento e empoderamento por parte dos profissionais (SANTOS RLN, et al., 2021).

A higienização das mãos é um dos hábitos mais importantes para evitar doenças e infecções, viabilizando a redução das Infecções Relacionadas à Assistência à Saúde (IRAS) para a menor taxa de incidência admissível e o baixo risco ou dano dispensável associados à atenção à saúde (OLIVEIRA FF e HONORATO $K A, 2021)$.

As atividades educativas sobre Paramentação e Desparamentação de Equipamentos de Proteção Individual (EPI), tem por objetivo proteger o trabalhador sempre que este estiver em contato com materiais biológicos durante a assistência cotidiana, praticando as precauções universais (SILVA GPC, et al., 2020).

O estudo de Ferreira CHP, et al. 2020), corrobora com os achados dessa pesquisa pois esses autores afirmam que a implementação de uma estação de treinamento diário em local pré-estabelecido e com horário fixo, possibilita a oportunidade de praticar sob a orientação e o olhar atento dos multiplicadores responsáveis por aquele turno ou setor.

A simulação clínica (por meio do realismo) oportuniza aos profissionais e/ou discentes realizar procedimentos assistenciais com segurança e possibilita também a formação de profissionais mais capacitados e confiantes na assistência ao paciente com covid-19, quando este estiver em ambiente real, desta forma contribui para minimização de riscos relacionados à assistência, além disso, a vivencia em um ambiente de simulação clínica estimula o profissional a tomar decisões perante a equipe de Enfermagem (PIMENTÃo RA, et al., 2021). 
Uso das tecnologias digitais da informação e da comunicação (TDICs), tem como principais objetivos promover aprendizagens significativas por se tratar de um conjunto de recursos tecnológicos que possibilita a interação entre os indivíduos envolvidos, além de promover a partilha do conhecimento desejado (SERRAVALLE LMK, et al., 2021).

Para Neto JBSB, et al. (2020), a elaboração de cartilha e vídeos informativos, a Educação em Saúde por meio da Tecnologia Educacional é uma ferramenta eficaz e valiosa em situações alarmantes, como a qual enfrentamos.

Metodologia ativa: Simulação realística de atendimento inicial ao paciente grave, suspeito ou confirmado de covid-19. A inclusão de metodologias ativas pode ampliar a visão dos profissionais e devem ser utilizadas em capacitações de outros momentos a fim de desenvolver habilidades que respondam às suas necessidades e consolidar o ensino continuado (ZINGRA KN, et al., 2020).

De acordo com o estudo de Santos RLN, et al. (2021), na execução dos treinamentos diante de uma pandemia houve um fortalecimento da Educação Permanente dentro das instituições de saúde, tornando-se mais visível para todas as áreas e não apenas para a área de enfermagem, havendo uma procura de outras áreas pela capacitação, fato este que antes acontecia de forma pontual.

Neste momento de pandemia da COVID-19, é importante compreender a realidade que os profissionais de saúde estão vivenciando, devido às demandas de atendimento integral e multidisciplinar. Os profissionais são convidados a refletir sobre as limitações e complexidades da prestação de cuidados, colocando-os diante de dois fenômenos já vivenciados historicamente na saúde: o medo da auto contaminação e da morte. Enfrentar uma pandemia de grandes proporções requer políticas de saúde abrangentes, e uma estrutura hospitalar robusta integrada ao sistema de saúde (BRANCO AO e TAVARES MMC, 2020).

A EPS surge como uma potencial linha educacional com estratégias relacionadas a mecanismos e temas que geram reflexões acerca do processo de trabalho, da autogestão, das mudanças institucionais e das transformações das práticas profissionais em diversos serviços. Essa política se configura através da proposta do aprender continuamente, do trabalhar em equipe, da construção de cotidianos e da apropriação do ser como objeto de aprendizagem individual, coletiva e institucional (MINISTÉRIO DA SAÚDE, 2018).

\section{CONSIDERAÇÕES FINAIS}

A alta transmissibilidade da SARS-CoV-2 apresenta-se como um desafio para a saúde pública mundial, com significativos impactos socioeconômicos e no sistema de saúde, diante deste cenário pandêmico a Educação Permanente em Saúde (EPS) é uma ferramenta imprescindível no enfrentamento a pandemia da COVID-19 tendo como objetivos promover aprendizagens significativas e críticas com possibilidades de transformar as práticas profissionais, apresenta-se como estratégias que visa promover e estabelecer as boas práticas nos serviços de saúde em vários níveis de atuação e complexidade, promovendo um ambiente de práticas seguras e contínuas na assistência.

\section{AGRADECIMENTOS E FINANCIAMENTO}

Agradecemos a bolsa concedida para autora Giselda Bezerra Correia Neves - Ao "Ministério da Ciência, Tecnologia, Inovações e Comunicações" (MCTIC). Ao "Conselho Nacional de Desenvolvimento Científico e Tecnológico" (CNPQ). Bolsista Industrial Tecnológico DTI-A CNPq, pesquisadora do ministério da educação. Número de concessão da bolsa: 304424/2020-0, Modalidade: Desenvolvimento Tecnológico Industrial - DTIA.

\section{REFERÊNCIAS}

1. BARBOSA SIM, MOREIRA FJF. Educação permanente em saúde como ferramenta de gestão para a atenção primária do SUS: a experiência do município de Caucaia - Ceará. Fortaleza. 20 ed. Escola de Saude publica do Ceará, 2019; 447p.

2. BRANCO AO, TAVARES MMC. Análise do processo de implantação do serviço de assistência ao paciente com COVID-19. Revista Online Braz J Nur, 2020; 19(3). 
3. FERREIRA CHP, et al. Estratégias adotadas no enfrentamento da covid-19: relato de experiência profissional. Revista Enfermagem em Foco, 2020; 11 (Esp.2): 199-204.

4. GEREMIA SD, et al. 200 Anos de Florence e os desafios da gestão das práticas de enfermagem na pandemia COVID19. Revista Latino-Americana de Enfermagem, 2020; 28:e3358

5. GONÇALVES SO, et al. Programa de educação permanente em saúde e a praxis profissional: possibilidades e desafios. Revista Eletrônica Acervo Saúde, 2020; 12(10): e4084.

6. JUNIOR AMF, et al. COVID-19 em profissionais da saúde, vivências e perspectivas: um relato de experiência. Revista Eletrônica Acervo Saúde, 2020; 12(12): e5258.

7. LOPES RSTM, et al. Educação permanente e humanização na transformação das práticas na atenção básica. Revista Mineira de Enfermagem, 2019; 23: e-1161

8. MARTINS AB, et al. A assistência multiprofissional a pacientes em tratamento de COVID-19 e a minimização do distanciamento familiar em um serviço de pronto atendimento em Manaus, Amazonas. Revista Eletrônica Acervo Saúde, 2020; 12(12): e5086.

9. MINISTÉRIO DA SAÚDE. Centro de Estudos e Pesquisas em Emergências e Desastres em Saúde (CEPEDES). Escola Nacional de Saúde Pública (ENSP). A gestão de riscos e governança na pandemia por COVID-19 no Brasil análise dos decretos estaduais no primeiro mês relatório técnico e sumário executivo, Brasil, 2020. 78p.

10. MINISTÉRIO DA SAÚDE. Secretaria de Atenção à Saúde. Política Nacional de Humanização - HumanizaSUS: Brasília: $\quad$ Brasil, $2013 . \quad 16 \mathrm{p}$ Disponível https://bvsms.saude.gov.br/bvs/publicacoes/politica_nacional_humanizacao_pnh_folheto.pdf. Acesso em: 02 setembro. 2021.

11. MINISTÉRIO DA SAÚDE. Secretaria de Gestão do Trabalho e da Educação na Saúde. Departamento de Gestão do Trabalho em Saúde. Cuidados no ambiente de assistência hospitalar ao paciente com suspeita ou diagnóstico de covid-19 - 1.ed. Brasília, DF: Brasil, 2020. 62p. Disponível em: http://conteudosdigitais.eerp.usp.br/covid19/cuidados_covid_ms_05_05_2020.pdf. Acesso em: 02 setembro. 2021.

12. MINISTÉRIO DA SAÚDE. Secretaria de Gestão do Trabalho e da Educação na Saúde. Departamento de Gestão da Educação na Saúde. Política Nacional de Educação Permanente em Saúde: o que se tem produzido para o seu fortalecimento? / Ministério da Saúde, Secretaria de Gestão do Trabalho e da Educação na Saúde, Departamento de Gestão da Educação na Saúde - 1. ed. rev. - Brasília: Brasil, 2018. 78p.

13. MINISTÉRIO DA SAÚDE. Secretaria de Vigilância em Saúde. Departamento de Análise em Saúde e Doenças não Transmissíveis. Guia de vigilância epidemiológica Emergência de saúde pública de Importância nacional pela Doença pelo coronavírus 2019 - covid-19 - Brasília: Brasil, 2021. Disponível em: https://www.conasems.org.br/wpcontent/uploads/2021/03/Guia-de-vigila\%CC\%82ncia-_ epidemiolo\%CC\%81gica-da-covid_19_15.03_2021.pdf. Acesso em: 02 setembro. 2021.

14. MONTALVÃO SA, et al. 2020. A EPS e a humanização no enfrentamento à covid-19 "produzindo redes e territórios vivos". Secretaria de Estado da Saúde, 2020; 58 p.

15. MOTTA DL, et al. COvid-19 evidências para todos: desenvolvimento de um objeto de aprendizagem no ensino em saúde. Revista Gaúcha de Enfermagem, 2021; 42(esp): e20200281

16. NETO JBSB, et al. Construção de tecnologias educativas como forma de educação em saúde para a prevenção da Covid-19: relato de experiência. Revista Eletrônica Acervo Saúde, 2020; 12(9): e3737.

17. OLIVEIRA FF, HONORATO KA. Atividade lúdica e educativa para higienização das mãos em tempos de

18. pandemia: relato de experiência. Revista Nursing, 2021; 24(275): 5496-5500.

19. PIMENTÃO RA, et al. Simulação clínica para enfrentamento da covid-19: treinamento complementar de enfermeiros. Revista de Enfermagem UFPE on line, 2021; 15: e246653

20. RIOS MFA, et al. Atenção primária à saúde frente à covid-19 em um centro de saúde. Revista Enfermagem em Foco, 2020; 11(1) Especial: 246-251.

21. SANTOS RLN, et al. Potencialidade da Educação Permanente na prevenção da infecção pelo Covid-19 em profissionais de saúde: relato de experiência. Revista Eletrônica Acervo Saúde, 2021; 13(3): e6465.

22. SERRAVALLE LMK, et al. Incorporação de tecnologias digitais nas estratégias de apoio à renast-ba durante a pandemia da covid-19. Revista Baiana de Saúde Pública, 2021; 45 (Esp.1): 267-281.

23. SILVA JF, et al. Educação permanente em saúde no contexto de uma unidade de saúde da família no município do Rio de Janeiro, Brasil. Revista Eletrônica Acervo Saúde, 2020; 12(9): e4327.

24. SILVA GPC, et al. Atividades educativas para uso adequado de equipamentos de proteção individual em hospital federal de referência. Revista Enfermagem em Foco, 2020; 11(1) Especial: 228-233.

25. ZINGRA KN, et al. Educação permanente para profissionais da área da saúde como estratégia de combate ao enfretamento da pandemia de COVID-19 na região norte: relato de experiência. Revista Eletrônica Acervo Saúde, 2020; 12(12): e5745. 